\title{
Pilot symbol assisted channel estimation for OFDM-based cognitive radio systems
}

\author{
Emmanuel Manasseh*, Shuichi Ohno and Masayoshi Nakamoto
}

\begin{abstract}
In this article, challenges regarding the provision of channel state information (CSI) in non-contiguous orthogonal frequency division multiplexing (NC-OFDM) cognitive radio (CR) systems are addressed. We propose a novel scheme that utilizes cross entropy (CE) optimization together with an analytical pilot power distribution technique to design pilot symbols that minimizes the channel estimate mean squared error (MSE) of frequency-selective channels. The optimal selection of pilot subcarriers is a combinatorial problem that requires heavy computations. To reduce the computational complexity, the CE optimization is utilized to determine the position of pilot subcarriers. Then, for a given pilot placement obtained by the CE algorithm, a closed form expression to obtain optimal pilot power distribution is employed. Simulation results indicate that, the proposed pilot symbol design provides better channel estimate MSE as well as the bit error rate (BER) performance when compared with the conventional equal powered pilot design.
\end{abstract}

Keywords: Cognitive radio, Cross entropy optimization, MSE, NC-OFDM

\section{Introduction}

The immense growth of wireless access technologies calls for more and more spectrum resources following the conventional spectrum framework, where most of the spectrum bands are exclusively allocated to specific licensed services. However, measurements have shown that, a lot of licensed bands are underutilized [1-3], which results into spectrum wastage and promotes artificial spectrum scarcity. This has fostered the spectrum regulatory bodies such as Federal Communications Commission (FCC) to open the licensed bands to unlicensed users through the use of cognitive radio $(\mathrm{CR})$ technology $[4,5]$.

To exploit limited spectrum efficiently, CR technology allows unlicensed users to access licensed spectrum bands. CR improves spectral efficiency by sensing the spectrum, detects the presence of the primary users (PUs) and exploits the unused spectrum without disturbing the PUs [3]. Since PUs have priorities to use the spectrum when the cognitive secondary users (SUs) co-exist with them, then SUs need to continuously monitor the events of the PUs to avoid interference and collisions. The

*Correspondence: manassehjc@hiroshima-u.ac.jp

Department of Artificial Complex Systems Engineering, Hiroshima University, 1-4-1 Kagamiyama Higashi-Hiroshima, 739-8527, Japan reliable results of the PUs' events are obtained through spectrum sensing, then based on the sensing results, the SUs can obtain information about the available spectrum that they can access [5].

The non-contiguous orthogonal frequency division multiplexing (NC-OFDM) transceivers are prominent candidates for $\mathrm{CR}$ systems as they are designed to transmit information in the presence of PUs. By deactivating subcarriers utilized by the PUs, interference between the PUs and the SUs can be mitigated [6,7]. However, the presence of deactivated subcarriers in the active subcarrier zone may possibly lead to non-contiguous sequence of the available subcarriers for the SUs and thereby complicate the design of efficient pilot symbols for channel estimations [6-9].

In the literature, several pilot symbols designs for channel estimation have been predominantly developed for OFDM systems with and without null edge subcarriers (see [10-16], and the references therein). Optimal pilot symbols for OFDM systems in the absence of null edge subcarriers are considered in [14-16] where equal distance and equal powered pilot symbols were found to be optimal with respect to several performance measures. However, in [10-13], it has been demonstrated that,

\section{是 Springer}

(c) 2013 Manasseh et al.: licensee Springer. This is an Open Access article distributed under the terms of the Creative Commons Attribution License (http://creativecommons.org/licenses/by/2.0), which permits unrestricted use, distribution, and reproduction in any medium, provided the original work is properly cited. 
for OFDM systems with null edge subcarriers equal distance and equal powered pilot symbols are not necessarily optimal.

The conventional pilot symbols designs in [10-13] are effective for traditional OFDM systems where the spectrum is contiguous except for the DC and null edge subcarriers. But they are no longer effective for NCOFDM systems. For an arbitrary set of pilot subcarriers, the methods in [10-13] can be adopted for pilot power distribution. However, the pilot positions optimization schemes (i.e., algorithms for determining the optimal pilot placements) are not effective for NC-OFDM systems.

In $[7,8]$, a scheme for designing pilot symbols for OFDM-based CR systems is proposed. The method in $[7,8]$ formulates the pilot design as an optimization problem that minimizes the upper bound related to the least square (LS) channel estimate mean squared error (MSE). An efficient scheme to solve the optimization problem is also proposed. The algorithm in $[7,8]$ considers equal powered pilot symbols and obtain the optimal placement of pilot symbols for a random set of activated subcarriers. However, the optimality of equal powered pilot symbols does not necessarily hold true when there are null subcarriers [10].

In this article, we propose a novel method that utilizes cross entropy (CE) optimization together with the analytical pilot power optimization to design pilot symbols for NC-OFDM to lower the MSE of the LS channel estimate of the frequency-selective channel. We formulate the pilot placement problem as a combinatorial problem and employ the CE optimization to select optimal placement of the pilot symbols. To the generated samples of pilot placements, we adopt the analytical power optimization for pilot power distribution in order to reduce the channel estimate MSE. Design examples consistent with IEEE 802.16e are provided to corroborate the superior performance of our proposed method over the equal powered pilot symbols in $[7,8]$.

The rest of this article is organized as follows. Section 2 introduces the system model for the NC-OFDM transceiver. In Section 3, the least square estimate is concisely described while in Section 4, pilot design for channel estimation is described. Simulation results are provided in Section 5 and finally, Section 6 concludes our design.

\section{System model}

We consider point-to-point wireless non-contiguous orthogonal frequency division multiplexing (NC-OFDM) transmissions over frequency-selective fading channels. We assume that the discrete-time baseband equivalent channel has a finite impulse response (FIR) of maximum length $L$, and remains constant in at least one OFDM symbol, i.e., is quasi-static. The channel impulse response is denoted as $\left\{h_{0}, h_{1}, \ldots, h_{L-1}\right\}$.

Let us consider the transmission of one OFDM symbol with $N$ number of subcarriers. In the NC-OFDM transceiver, not all subcarriers are active as in conventional OFDM transmission. The active subcarriers are located in the unoccupied spectrum bands determined by spectrum sensing techniques.

At the transmitter, a symbol sequence $\left\{X_{0}, X_{1}, \ldots\right.$, $X_{N-1}$ \} undergoes serial-to-parallel (S/P) conversion to be stacked into one OFDM symbol. Then, an $N$-points inverse discrete Fourier transform (IDFT) follows to produce the $N$ dimensional data, which is parallel-to-serial $(\mathrm{P} / \mathrm{S})$ converted. The discrete-time baseband NC-OFDM signal, consisting of all $N$ contiguous subcarriers can be expressed in the time-domain as

$$
s_{n}=\frac{1}{\sqrt{N}} \sum_{k=0}^{N-1} X_{k} e^{j \frac{2 \pi k n}{N}}, \quad n \in[0, N-1],
$$

where the symbol over the $k$ th deactivated subcarriers is $X_{k}=0$.

Prior to transmission, a guard interval also known as a cyclic prefix (CP) is appended to each OFDM symbol to mitigate multipath effects. We assume that the length of the cyclic prefix $N_{c p}$, is greater than the channel length $L$ so that there is no inter-symbol interference (ISI) between consecutive OFDM symbols. At the receiver, we assume perfect timing and frequency synchronization. After removing $\mathrm{CP}$, we apply discrete Fourier transform (DFT) to the received time-domain signal $y_{n}$ for $n \in$ $[0, N-1]$ to obtain for $k \in[0, N-1]$

$$
Y_{k}=\frac{1}{\sqrt{N}} \sum_{n=0}^{N-1} y_{n} e^{-j \frac{2 \pi k n}{N}}=H_{k} s_{k}+W_{k},
$$

where $H_{k}$ is the channel frequency response at frequency $2 \pi k / N$ given by

$$
H_{k}=\sum_{l=0}^{L-1} h_{l} e^{-j \frac{2 \pi k l}{N}},
$$

and the noise $W_{k}$ is assumed to be i.i.d. circular Gaussian with zero mean and variance $\sigma_{w}^{2}$.

Since for OFDM-based cognitive radio (CR) systems, subcarriers occupied by the primary users (PUs) are deactivated, the cognitive secondary user (SU) utilizes only the activated subcarriers for transmission of data signals and pilot symbols. Thus, to ensure correct demodulation of data over the activated subcarriers, information about the activated subcarriers can be transmitted to the receiver via control channel before any data communication process begins. 
Let $\mathcal{K}$ be a set of activated subcarriers, then cardinality of the set $\mathcal{K}$ can be represented as $|\mathcal{K}|$. For channel estimation, we place $N_{p}(\leq|\mathcal{K}|)$ pilot symbols $\left\{p_{1}, \ldots, p_{N_{p}}\right\}$ at subcarriers $k_{1}, k_{2}, \cdots, k_{N_{p}} \in \mathcal{K}\left(k_{1}<k_{2}<\cdots<\right.$ $\left.k_{N_{p}}\right)$, which are known at the receiver. The number of pilot symbols affects not only on the channel estimation accuracy but also on the data rate. However, it is not easy to analyze its impact on the overall system performance in terms of data rate, since it depends on many factors. Here we just assume that $N_{p} \geq L$ so that the channel can be perfectly estimated if there is no noise. Let us denote the index of pilot symbols as $\mathcal{K}_{p}=\left\{k_{1}, \ldots, k_{N_{P}}\right\}$ and index of the SU data carrying subcarriers as $\mathcal{K}_{d}$, that is, $\mathcal{K}_{d} \subseteq \mathcal{K} \backslash \mathcal{K}_{p}$ where $\backslash$ denotes set difference. The number of data subcarriers is $N_{d}=\left|\mathcal{K}_{d}\right|$.

Let $\operatorname{diag}(\boldsymbol{a})$ be a diagonal matrix with the vector $\boldsymbol{a}$ on its main diagonal. Collecting the received signals having pilot symbols as $\tilde{\boldsymbol{Y}}=\left[Y_{k_{1}}, \ldots, Y_{k_{N_{p}}}\right]^{T}$, we obtain

$$
\tilde{\boldsymbol{Y}}=\boldsymbol{D}_{H_{p}} \boldsymbol{p}+\tilde{\boldsymbol{W}}
$$

where $\boldsymbol{D}_{H_{p}}$ is a diagonal matrix with its $n$th diagonal entry being $H_{k_{n}}$ such that $\boldsymbol{D}_{H_{p}}=\operatorname{diag}\left(H_{k_{1}}, \ldots, H_{k_{N_{p}}}\right)$, and $\boldsymbol{p}$ is a pilot vector defined as $\boldsymbol{p}=\left[p_{1}, \ldots, p_{N_{p}}\right]^{T}$, the i.i.d. Gaussian noise vector $\tilde{W}$ is defined as $\tilde{W}=$ $\left[W_{k_{1}}, \ldots, W_{k_{N_{p}}}\right]^{T}$.

From $\tilde{\boldsymbol{Y}}$, we would like to estimate channel frequency responses for equalization and decoding. Thus, $H_{k}$ for $k \in \mathcal{K}$ have to be estimated from $\tilde{\boldsymbol{Y}}$. In pilot-symbolassisted modulation (PSAM) [10-13], a few known pilot symbols are embedded in an OFDM symbol to facilitate the estimation of the unknown channel.

\section{Least square channel estimation}

We define $\boldsymbol{F}$ as an $N \times N$ DFT matrix, whose $(m+1$, $n+1)$ th entry is $e^{-j 2 \pi m n / N}$ for $m, n \in[0, N-1]$. We denote an $N \times L$ matrix $\boldsymbol{F}_{L}=\left[\boldsymbol{f}_{0}, \ldots, \boldsymbol{f}_{N-1}\right]^{\mathcal{H}}$ consisting of $N$ rows and the first $L$ columns of the DFT matrix $\boldsymbol{F}$, where $\mathcal{H}$ is the complex conjugate transpose operator. We also define an $N_{p} \times L$ matrix $\boldsymbol{F}_{p}$ having $\boldsymbol{f}_{k_{n}}^{\mathcal{H}}$ for $k_{n} \in \mathcal{K}_{p}$ as its $n$th row. Then, we can express (4) as

$$
\tilde{\boldsymbol{Y}}=\boldsymbol{D}_{p} \boldsymbol{F}_{p} \boldsymbol{h}+\tilde{\boldsymbol{W}}
$$

where the diagonal matrix $\boldsymbol{D}_{p}$ and channel vector $\boldsymbol{h}$ are respectively defined as $\boldsymbol{D}_{p}=\operatorname{diag}\left(p_{1}, \ldots, p_{N_{p}}\right)$, and $\boldsymbol{h}=$ $\left[h_{0}, \ldots, h_{L-1}\right]^{T}$.

Let a vector having channel responses to be estimated, i.e., $H_{k}$ for $k \in \mathcal{K}_{d}$, be $\boldsymbol{H}_{d}=\left[H_{k_{1}}, \ldots, H_{k_{\left|\mathcal{K}_{d}\right|}}\right]^{T}$. Similar to $\boldsymbol{F}_{p}$, we define a $\left|\mathcal{K}_{d}\right| \times L$ matrix $\boldsymbol{F}_{d}$ having $\boldsymbol{f}_{k_{n}}^{\mathcal{H}}$ for $k_{n} \in \mathcal{K}_{d}$ as its $n$th row, where $k_{n}<k_{n^{\prime}}$ if $n<n^{\prime}$. Then, we obtain

$$
\boldsymbol{H}_{d}=\boldsymbol{F}_{d} \boldsymbol{h}
$$

Since (5) is linear, the least squares (LS) estimate $\hat{\boldsymbol{H}}_{d}$ of $\boldsymbol{H}_{d}$ is given by

$$
\hat{\boldsymbol{H}}_{d}=\boldsymbol{F}_{d}\left(\boldsymbol{D}_{p} \boldsymbol{F}_{p}\right)^{\dagger} \tilde{\boldsymbol{Y}}
$$

where $(\cdot)^{\dagger}$ stands for the Moore-Penrose pseudo-inverse of a matrix. The LS estimate does not require any prior knowledge on channel statistics and is thus widely applicable.

If we define the estimation error vector $\boldsymbol{E}_{d}=\hat{\boldsymbol{H}}_{d}-\boldsymbol{H}_{d}$, then the correlation matrix $\boldsymbol{R}_{e}$ of $\boldsymbol{E}_{d}$ can be expressed as $[10,12]$

$$
\boldsymbol{R}_{e}=E\left\{\boldsymbol{E}_{d} \boldsymbol{E}_{d}^{\mathcal{H}}\right\}=\boldsymbol{F}_{d}\left[\frac{1}{\sigma_{w}^{2}} \boldsymbol{F}_{p}^{\mathcal{H}} \boldsymbol{\Lambda}_{p} \boldsymbol{F}_{p}\right]^{-1} \boldsymbol{F}_{d}^{\mathcal{H}},
$$

where $E\{\cdot\}$ stands for the expectation operator and $\boldsymbol{\Lambda}_{p}$ is a diagonal matrix given by

$$
\boldsymbol{\Lambda}_{p}=\boldsymbol{D}_{p}^{\mathcal{H}} \boldsymbol{D}_{p}=\operatorname{diag}\left(\lambda_{1}, \ldots, \lambda_{N_{p}}\right)
$$

with $\lambda_{n}=\left|p_{k_{n}}\right|^{2}$ for $k_{n} \in \mathcal{K}_{p}$.

For a traditional OFDM symbol without null subcarriers, $\boldsymbol{F}_{d}^{\mathcal{H}} \boldsymbol{F}_{d}=\boldsymbol{c I}$ for a non-zero constant $c$, then $E\left\{\left\|\boldsymbol{E}_{d}\right\|^{2}\right\}=c E\left\{\|\hat{\boldsymbol{h}}-\boldsymbol{h}\|^{2}\right\}$, where $\|\cdot\|$ denotes the Euclidean norm. The Equation $\boldsymbol{F}_{d}^{\mathcal{H}} \boldsymbol{F}_{d}=c \boldsymbol{I}$ is attained if all pilot symbols have the same power and are uniformly distributed in an OFDM symbol. But, this is not always possible if there are null subcarriers in the OFDM symbol $[10,12]$. Thus, for NC-OFDM equal powered pilot symbols are not necessarily optimal.

Now, our objective is to find the optimal pilot symbols that minimize the channel estimate MSE at the activated subcarriers, which is defined as

$$
\eta=\left(\sum_{k \in \mathcal{K}} E\left\{\left|\hat{H}_{k}-H_{k}\right|^{2}\right\}\right)^{\frac{1}{2}}=\left(\operatorname{trace} \boldsymbol{R}_{e}\right)^{\frac{1}{2}} .
$$

Note that, $\eta^{2}=E\left\{\left\|\hat{\boldsymbol{H}}_{d}-\boldsymbol{H}_{d}\right\|^{2}\right\} \neq c E\left\{\|\hat{\boldsymbol{h}}-\boldsymbol{h}\|^{2}\right\}$ if $\boldsymbol{F}_{d}^{\mathcal{H}} \boldsymbol{F}_{d} \neq c \boldsymbol{I}$.

\section{Design of pilot symbols for NC-OFDM}

In this section, we consider pilot-symbol-assisted transmission where periodically embedded pilot symbols, known by both the transmitter and the receiver are used to estimate the fading channel coefficients of the timevarying channel. For a given $\mathcal{K}$, we propose a cross entropy (CE) based algorithm together with analytical pilot power distribution technique to determine pilot set $\mathcal{K}_{p}$ with a potential of minimizing the channel estimate mean square error (MSE).

Our objective is to find the optimal pilot symbols that minimize the channel estimate MSE at the activated data subcarriers. The design of optimal pilot symbol calls for joint optimization of the pilot placement $\mathcal{K}_{p}$ and power 
distribution $\lambda$. Thus, the optimization problem can be expressed as

$$
\left(\lambda^{\star}, \mathcal{K}_{p}^{\star}\right)=\arg \min _{\lambda, \mathcal{K}_{p}} \eta^{2}
$$

under the constraints that $\mathcal{K}_{p} \subset \mathcal{K}$, and

$$
\lambda \succeq 0,
$$

where $\lambda=\left[\lambda_{1}, \ldots, \lambda_{N_{p}}\right]^{T}$, and $\sum_{k=1}^{N_{p}} \lambda_{k}=1$. The optimization problem in (11) can be reexpressed as

$$
\begin{aligned}
& \min _{\lambda, \mathcal{K}_{p}} \sigma_{w}^{2} \text { trace }\left[\boldsymbol{F}_{d}\left(\boldsymbol{F}_{p}^{\mathcal{H}} \boldsymbol{\Lambda}_{p} \boldsymbol{F}_{p}\right)^{-1} \boldsymbol{F}_{d}^{\mathcal{H}},\right] \\
& \text { subject to } \lambda \succeq 0, \quad \mathcal{K}_{p} \subset \mathcal{K} .
\end{aligned}
$$

The optimization problem in (11) is analytically intractable as it requires the joint optimization of pilot indices $\mathcal{K}_{p}$ and pilot power distribution $\lambda$. Numerical optimization is possible but computational inefficient because power distribution to the pilot symbols is not uniform. In $[7,8]$, similar optimization is considered with equal powered pilot symbols assumed to be optimal, thereby optimizing only the indices of the pilot symbols. By employing equal powered pilot symbols, the optimization problem in $[7,8]$ is simplified. However, for NC-OFDM equal power pilot symbols are not necessarily optimal.

To determine the optimal set $\mathcal{K}_{p}$, i.e., the optimal location of $N_{p}$ pilot symbols, we have to enumerate all possible sets, then optimize the pilot symbols for each set and compare them. This design approach becomes intractable as $|\mathcal{K}|$ gets larger.

In the literature, several randomized optimization algorithms based on the $\mathrm{CE}$ method have been proposed and have been shown to lead to good performances on numerous optimization problems [17-19], often outperforming other randomized algorithms [18]. Motivated by the effectiveness of the CE method for finding nearoptimal solutions in huge search spaces, this article adopts the CE-based method to search for the near-optimal position of the pilot symbols with lower least square (LS) channel estimate MSE. The CE method is an iterative procedure for evaluation of rare event probabilities and also for combinatorial optimizations. Each iteration involves generating a random sample according to a probability distribution and then updating the parameters of the probability distribution in order to produce better samples in the next iteration. While applications of the CE-based methods to various disciplines of engineering have already been reported (see, e.g. [19-22]), to the best of our knowledge, they have not been employed yet to pilot symbol designs for channel estimation in conventional as well as NC-OFDM.
Let us denote the set $\mathcal{K}_{p}^{m}$ as

$$
\mathcal{K}_{p}^{m}=\mathcal{K}\left(\left\{I_{k}\right\}_{k=1}^{|\mathcal{K}|}=1\right), \quad I_{k} \in\{0,1\}, \quad m=1, \ldots, M,
$$

where the indicator function $I_{k}$ shows whether a subcarrier at the $k$ th position is selected. The set of all $M=\left(\begin{array}{l}|\mathcal{K}| \\ \left|\mathcal{K}_{p}\right|\end{array}\right)$ possible subsets is denoted by $\boldsymbol{\Omega}=\left\{\mathcal{K}_{p}^{1}, \ldots, \mathcal{K}_{p}^{M}\right\}$, where $\left(\begin{array}{l}a \\ b\end{array}\right)$ denotes the possible combination set.

Once the pilot subcarriers are determined, we can apply the analytical method in [11], which employ the Lagrange multiplier to solve the optimization problem with respect to $\lambda$ under the power constraint. The optimal power distribution is given by [11]

$$
\lambda^{\star}(n)=\sigma_{w}^{2} \frac{\sqrt{\Phi(n, n)}}{\sum_{n=0}^{L} \sqrt{\Phi(n, n)}}, \quad n=0, \ldots, L-1
$$

where

$$
\Phi=\left(\boldsymbol{F}_{d} \boldsymbol{F}_{p}^{-1}\right)^{\mathcal{H}}\left(\boldsymbol{F}_{d} \boldsymbol{F}_{p}^{-1}\right) .
$$

Note that, Equation (15) is essentially the same as the analytical power distribution in [13] and the two designs give the same power distribution. Convex optimization designs in $[10,12]$ can also be used for power distribution, however the complexity of the convex optimization design is higher than the analytical power distribution. Moreover, convex optimization designs are not stable for OFDM systems with large number of subcarriers [13].

Our optimal pilot sequence design can be formulated as a combinatorial optimization problem as

$$
\mathcal{K}_{p}^{\star}=\arg \min _{\mathcal{K}_{p}^{m} \in \boldsymbol{\Omega}} \mathcal{C}_{\text {sel }}\left(\mathcal{K}_{p}^{m}\right),
$$

where

$$
\mathcal{C}_{\text {sel }}\left(\mathcal{K}_{p}^{m}\right)=\operatorname{trace}\left[\boldsymbol{F}_{d}\left(\boldsymbol{F}_{p}^{\mathcal{H}} \boldsymbol{\Lambda}_{p} \boldsymbol{F}_{p}\right)^{-1} \boldsymbol{F}_{d}^{\mathcal{H}}\right],
$$

represents the channel estimate MSE of the pilot set $\mathcal{K}_{p}^{m}$, and $\mathcal{K}_{p}^{\star}$ is the global optimal set of the objective function.

Using (15) and (16), the objective function can be represented as [13]

$$
\mathcal{C}_{\text {sel }}\left(\mathcal{K}_{p}^{m}\right)=\lambda_{p}^{-1} \operatorname{diag}\left(\boldsymbol{\Phi}^{\mathcal{H}} \boldsymbol{\Phi}\right)
$$

which is (19) is much simpler than (18).

Applying the CE to solve (17), the first step is transforming the deterministic optimization problem (17) into a family of stochastic sampling problems $[17,18]$. Since the considered problem is on a discrete case, a family of Bernoulli probability density functions associated with the pilot symbol selection vector, $\boldsymbol{\omega}=\left[\omega_{1}, \omega_{2}, \ldots, \omega_{|\mathcal{K}|}\right]$, $\omega_{k} \in\{0,1\}$, is given by

$$
f(\boldsymbol{\omega} ; \boldsymbol{q})=\prod_{k=1}^{|\mathcal{K}|} q_{k}^{[\omega]_{k}}\left(1-q_{k}^{1-[\omega]_{k}}\right)
$$


where $\boldsymbol{q}=\left[q_{1}, q_{2}, \ldots q_{|\mathcal{K}|}\right]$ is a probability vector whose $q_{k}$ entry indicates the probability of selecting the $k$ th subcarrier, and $[\omega]_{k} \in\{0,1\}$ indicates whether the $k$ th element of $\omega_{k}$ (the $k$ th tone) is selected. If $\omega_{k}$ is selected, then $[\omega]_{k}=1$. Each element of $\mathcal{K}_{p}^{m}$ is modeled as an independent Bernoulli random variable with probability mass function $q\left(\omega_{k}=1\right)=q_{k}$, and $q\left(\omega_{k}=0\right)=1-q_{k}$, for $k=1, \ldots,|\mathcal{K}|$.

The CE method aims to find an optimal probability distribution $\boldsymbol{q}^{\star}$ that generates an optimal solution $\omega^{\star}$ with minimum channel estimate MSE. However, $\omega^{\star}$ occurs with a very small probability. In this case, (17), is associated with the problem of estimating the probability $\operatorname{Pr}\left[\mathcal{C}_{\text {sel }}(\omega) \leq \gamma\right]$ for a given threshold $\gamma$. The associated stochastic estimation problem is

$$
\ell=\operatorname{Pr}\left[\mathcal{C}_{\text {sel }}(\boldsymbol{\omega}) \leq \gamma\right]=\sum_{u=1}^{\mathcal{U}} I_{\left\{\mathcal{C}_{\mathrm{sel}}\left(\boldsymbol{\omega}^{(u)}\right) \leq \gamma\right\}} f(\boldsymbol{\omega} ; \boldsymbol{q}),
$$

where $\mathcal{U}$ is a number of the generated samples (i.e., candidate solutions) and $\left\{\mathcal{C}_{\text {sel }}\left(\omega^{(t, u)}\right) \leq \gamma^{(t)}\right\}$ is a variable defined by

$$
I_{\left\{\mathcal{C}_{\text {sel }}\left(\omega^{(u)}\right) \leq \gamma\right\}}= \begin{cases}1, & \text { if } \mathcal{C}_{\text {sel }}\left(\omega^{(u)}\right) \leq \gamma \\ 0, & \text { otherwise }\end{cases}
$$

A simple way to estimate $\ell$ is via importance sampling (IS). Let us take a set of random samples $\mathcal{C}_{\text {sel }}^{1}, \mathcal{C}_{\text {sel }}^{2}, \ldots, \mathcal{C}_{\text {sel }}^{\mathcal{U}}$ drawn from the distribution $f(\omega ; v)$. Then, the likelihood ratio estimator of $\ell$ is [18]

$$
\hat{\ell}=\frac{1}{\mathcal{U}} \sum_{u=1}^{\mathcal{U}} I_{\left\{\mathcal{C}_{\mathrm{sel}}\left(\boldsymbol{\omega}^{(u)}\right)\right\}} \frac{f(\boldsymbol{\omega} ; \boldsymbol{q})}{f(\boldsymbol{\omega}, \boldsymbol{v})} .
$$

Note that, $f(\boldsymbol{\omega}, \boldsymbol{v})$ is chosen such that he cross entropy between $f(\boldsymbol{\omega}, \boldsymbol{v})$ and $f(\boldsymbol{\omega}, \boldsymbol{q})$ is minimal. Thus, an optimum parameter $\boldsymbol{q}$ can be found by minimizing the CE between the two distributions $f(\boldsymbol{\omega} ; \boldsymbol{q})$ and $f(\boldsymbol{\omega}, \boldsymbol{v})$. To minimize this Kullback-Leibler divergence is equivalent to solving the optimization problem in $[18,23]$

$$
\begin{aligned}
\min _{\boldsymbol{q}} \hat{D}(\boldsymbol{q}) & =\min _{\boldsymbol{q}} \frac{1}{\mathcal{U}} \sum_{u=1}^{\mathcal{U}} I_{\left\{\mathcal{C}_{\mathrm{sel}}\left(\boldsymbol{\omega}^{(u)}\right)\right\}} \log f(\boldsymbol{\omega} ; \boldsymbol{q}) \\
& =\min _{\boldsymbol{q}} \frac{1}{\mathcal{U}} \sum_{u=1}^{\mathcal{U}} I_{\left\{\mathcal{C}_{\mathrm{sel}}\left(\boldsymbol{\omega}^{(u)}\right)\right\}} \log \left(\prod_{k=1}^{|\mathcal{K}|} q_{k}^{[\boldsymbol{\omega}]_{k}}\left(1-q_{k}^{1-[\boldsymbol{\omega}]_{k}}\right)\right)
\end{aligned}
$$

where $\hat{D}$ is the likelihood ratio estimator of $D$, which is the Kullback-Leibler divergence. The optimal value of $\hat{D}(\boldsymbol{q})$ is obtained by setting $\partial \hat{D} / \partial \boldsymbol{q}=0$, hence the update rule is given by

$$
q_{k}=\frac{\sum_{u=1}^{\mathcal{U}} I_{\left\{\mathcal{C}_{\text {sel }}\left(\omega^{(u)}\right) \leq \gamma\right\}} I_{k}\left(\omega^{(u)}\right)}{\sum_{u=1}^{\mathcal{U}} I_{\left\{\mathcal{C}_{\text {sel }}\left(\omega^{(u)}\right) \leq \gamma\right\}}},
$$

To estimate the rare event, $\mathrm{CE}$ iteratively updates the probability vector $\boldsymbol{q}$ so that most samples generated by $f(\boldsymbol{\omega} ; \boldsymbol{q})$ satisfy $\mathcal{C}_{\text {sel }} \leq \gamma$. By iteratively improving $\gamma, f(\boldsymbol{\omega} ; \boldsymbol{q})$ eventually converges to an optimum probability density function $f\left(\boldsymbol{\omega} ; \boldsymbol{q}^{\star}\right)$ and optimal $\boldsymbol{\omega}^{\star}$ can be obtained from $\boldsymbol{q}^{\star}$ by $f\left(\boldsymbol{\omega} ; \boldsymbol{q}^{\star}\right)$.

A standard CE procedure for solving combinatorial problems contains two stages [17-19,23].

1. Adaptive updating of $\gamma^{t}$ : For a given $q^{t-1}$ generate $\mathcal{U}$ random samples $\left\{\boldsymbol{\omega}^{(t, u)}\right\}_{\mathcal{u}=1}^{\mathcal{U}}$ from $f\left(. ; \boldsymbol{q}^{t-1}\right)$, where $t$ denotes the iteration index of CE. Then, calculate the channel estimate MSE according to (17) to obtain a set of performance values $\left\{\mathcal{C}_{\text {sel }}\left(\omega^{(t, u)}\right)\right\}_{u=1}^{\mathcal{U}}$ and rank them in ascending order so that $\mathcal{C}_{\text {sel }}^{1} \leq \cdots \leq \mathcal{C}_{\text {sel }}^{\mathcal{U}}$. Finally, assign

$$
\gamma^{t}=\mathcal{C}_{\mathrm{sel}}^{\lceil\rho \mathcal{U}\rceil}
$$

where $\rho$ denotes the fraction of the best samples and $\lceil\cdot\rceil$ is the ceiling operation. For description on how to select a suitable value of $\mathcal{U}$ see [17].

2. Adaptive updating of $\boldsymbol{q}^{t}$ : For a given $\gamma^{(t)}$ and $\boldsymbol{q}^{(t-1)}$, use the same samples $\left\{\mathcal{C}_{\text {sel }}\left(\boldsymbol{\omega}^{(t, u)}\right)\right\}_{u=1}^{\mathcal{U}}$ to update the parameter $\boldsymbol{q}^{(t)}=\left[q_{0}^{(t)}, q_{1}^{(t)}, \ldots, q_{|\mathcal{K}|}^{(t)}\right]$ via (25)

Note that in order to prevent fast convergence to a local optimum, parameter $\boldsymbol{q}^{t-1}$ is not updated to $\boldsymbol{q}^{t}$ directly; a smoothing factor, $\alpha, 0 \leq \alpha \leq 1$ was suggested by $[17,19]$ to update (22) into

$$
\boldsymbol{q}^{t}=\alpha \times \boldsymbol{q}^{t}+(1-\alpha) \times \boldsymbol{q}^{t-1} .
$$

When $\alpha=1$ the original updating formulation is achieved.

Algorithm 1 summarizes our proposed design. $\mathcal{J}$ is the predefined total number of iteration and $\mathcal{Z}$ is number of iterations without improvement.

\section{Algorithm 1 Pilot symbol selection}

1. Initialize probability vector $\boldsymbol{q}^{(0)}=q_{1}^{(0)}, \ldots, q_{|\mathcal{K}|}^{(0)}$ with $q_{k}^{(0)}=0.5$.

2. Set the iteration counter $t=0$ and $t^{\prime}=0$

3. while $\left(t^{\prime}<\mathcal{Z}\right.$ and $\left.t<\mathcal{J}\right)$

Generate $\mathcal{U}$ random samples $\left\{\omega^{(t, u)}\right\}_{u=1}^{\mathcal{U}}$ from the density function $f\left(\boldsymbol{\omega} ; \boldsymbol{q}^{(t-1)}\right)$, under the constraints in (20).

5. Optimize power of the generated samples (pilot subcarriers set), using (15).

6. Compute the performance functions $\mathcal{C}_{\text {sel }}\left(\omega^{(t, u)}\right)$ for $u=1,2, \ldots, \mathcal{U}$, and rank them in ascending order so that $\mathcal{C}_{\text {sel }}^{1} \leq \mathcal{C}_{\text {sel }}^{2} \leq \ldots \leq \mathcal{C}_{\text {sel }}^{\mathcal{U}}$ and use (26) to find $\gamma^{t}$.

7. Calculate the parameter $\boldsymbol{q}^{(t)}$ using (25). 


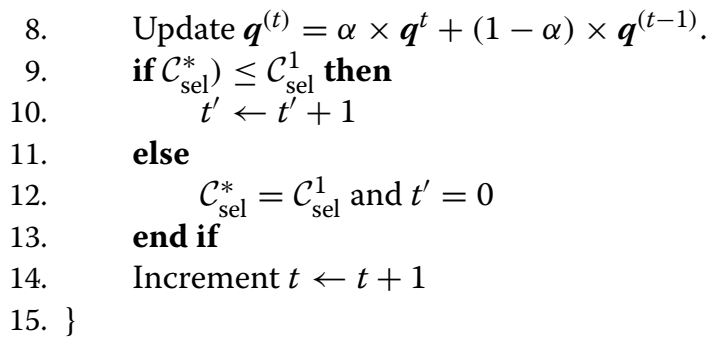

Note that, when convergence to degenerate value occurs, the algorithm can be stopped since it will only produce identical degenerate afterwards. CE-based algorithms can return the best answer possible even if they are not allowed to run to completion and may improve on the answer if they are allowed to run longer. Thus, for real time applications, introducing the parameter $\mathcal{Z}$ to control is number of iterations without improvement can significantly lower the computational time.

In general, the computational complexity of the CEbased method is relatively higher than that of the conventional reference design in $[7,8]$. However, with best selection of the CE parameters, the computational time can be reduced. Furthermore, the rapid growth of digital signal processing (DSP) and microprocessors could make the online implementation of the CE design practically feasible. Recently, a large number of digital signal processors (DSPs) and special microprocessors that can perform mathematical computations instantaneously with a high level of precision for real-time processing are available in the market. With these DSP circuits and microprocessors the CE-based schemes can be effectively and efficiently implemented in the real time environment.

The CE-based design can also be extended to design pilot symbols when multiple transmit antennas are deployed. In multiple input multiple output (MIMO) scenario where disjoint pilot symbols are utilized for each transmit antenna, the CE-based algorithm can efficiently design pilot placement for each antenna by adopting the algorithm in [24].

\subsection{Computational complexity analysis}

In this section, we evaluate the computational complexity of our proposed scheme over the exhaustive search (ES) and the conventional reference design in $[7,8]$. The complexity of the CE and ES algorithms is dominated by the computation of the analytical power distribution and the objective function. In general, the complexity of the CE scheme depends on the number of generated samples and the number of iterations. In our proposed design, for each iteration it is necessary to optimize power distribution of the generated samples as well as computing the performance or the objective function of all samples. Here, we are interested in bounding the number of iterations (steps) required to obtain the best performing samples.
For a given set of pilot subcarriers, Equation (15) requires $\mathcal{O}\left(N_{d} N_{p} L\right)$ operations to compute the power of the pilot symbol. Thus, optimizing power distribution to the $\mathcal{U}$ generated samples require $\mathcal{O}\left(\mathcal{U} N_{d} N_{p} L\right)$ operations, and the computational of the objective function in (19) for all generated samples require $\mathcal{O}\left(\mathcal{U} N_{p} L\right)$ operations. Note that, $\boldsymbol{\Phi}$ is a diagonal matrix already computed in (15), thus the complexity of (19) is relatively low because it utilizes the predetermined parameters. Except for the initialization, adaptive update of the probability function in (25) requires $\mathcal{O}\left(\mathcal{U} N_{p}\right)$ operations. Thus one iteration of a CE algorithm requires $\mathcal{O}\left(\left[N_{d} N_{p} L+N_{p} L+N_{p}\right] \mathcal{U}\right)$ operations. As it can be seen, the computational complexity of the algorithm is mainly due to the calculation of power and the objective function.

In practical application, specialized DSP microprocessors can be implemented to perform the power distribution and MSE computations easily. Furthermore, in our proposed scheme, the computational complexity can be reduced by careful selection of $\mathcal{U}$ and the total number of iterations $\mathcal{J}$.

ES scheme involves optimization of power and computation of the performance function to all possible samples. The complexity of the ES algorithm is $\mathcal{O}\left(\left[N_{d} N_{p} L+\right.\right.$ $\left.N_{p} L\right]\left(\begin{array}{l}|\mathcal{K}| \\ N_{p}\end{array}\right)$ ), which is relatively higher compared to that of the CE scheme. The scheme inn $[7,8]$ does not optimize power of the pilot symbols, and most of the parameters used for evaluating the pilot placement are predetermined, thereby reducing the computational complexity of the design.

\section{Simulation results}

In this section, we demonstrate the effectiveness of our proposed pilot design through computer simulations. The parameters of the transmitted OFDM signal studied in our design examples are as in the IEEE 802.16e and the IEEE 802.16m standards. For IEEE 802.16e, an OFDM transmission frame with $N=256$ is considered. Out of 256 subcarriers, $N_{a}=200$ are used for data and pilots (data carrying subcarriers). Of the remaining 56 subcarriers, 28 are null in the lower frequency guard band while 27 are nulled in the upper frequency guard band and one is the central DC null subcarrier ([25],p. 429).

For IEEE $802.16 \mathrm{~m}$, we consider an OFDM frame with $N=1024$, where $N_{a}=864$ are data carrying subcarriers and the remaining subcarriers are lower and upper frequency guard band subcarriers except for the DC subcarrier ([26], p. 537).

Of the subcarriers used for signal transmission (i.e., data carrying subcarriers), a set of $\mathcal{K}$ subcarriers used by the cognitive secondary user (SU) is generated randomly as in [6]. We define the ratio between the number of subcarriers used by the cognitive SUs $|\mathcal{K}|$ over the total number of 


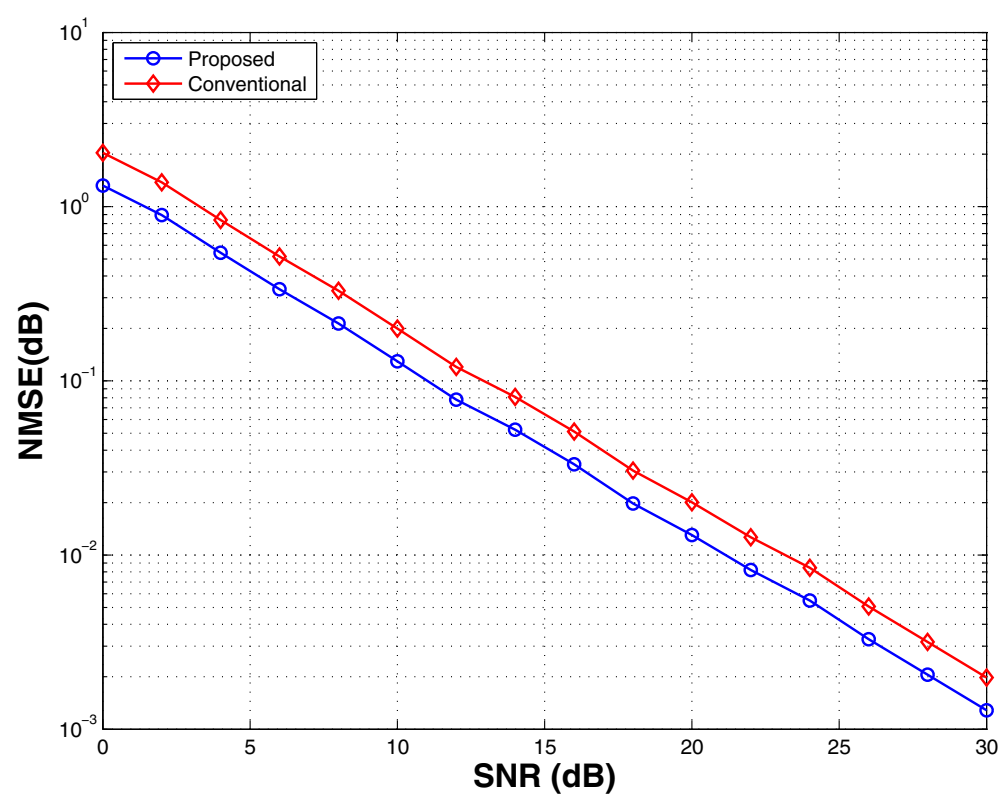

Figure 1 Performance of the channel estimate MSE for different SNRs, $L=N_{p}=8$.

data carrying subcarriers $N_{a}$ as $\beta=\frac{|\mathcal{K}|}{N_{a}}$. The performance of the system is measured in terms of the MSE and the bit error rate (BER) for the zero forcing (ZF) equalizer for different proportions of $|\mathcal{K}|$ and $N_{a}$.

Figure 1 shows the MSE of the channel estimator against signal to noise ratio (SNR) for the proposed scheme and the pilot symbols in $[7,8]$ which we refer to it as a conventional method for $L=N_{p}=8$ and $N=256$. From the result it is clear that, the proposed design outperforms the conventional method by a constant gap for different SNRs. This may be due to the fact that the conventional design consider equal powered pilots and optimizes only the position. To ensure better MSE performance, both pilot position and power distribution need to be careful

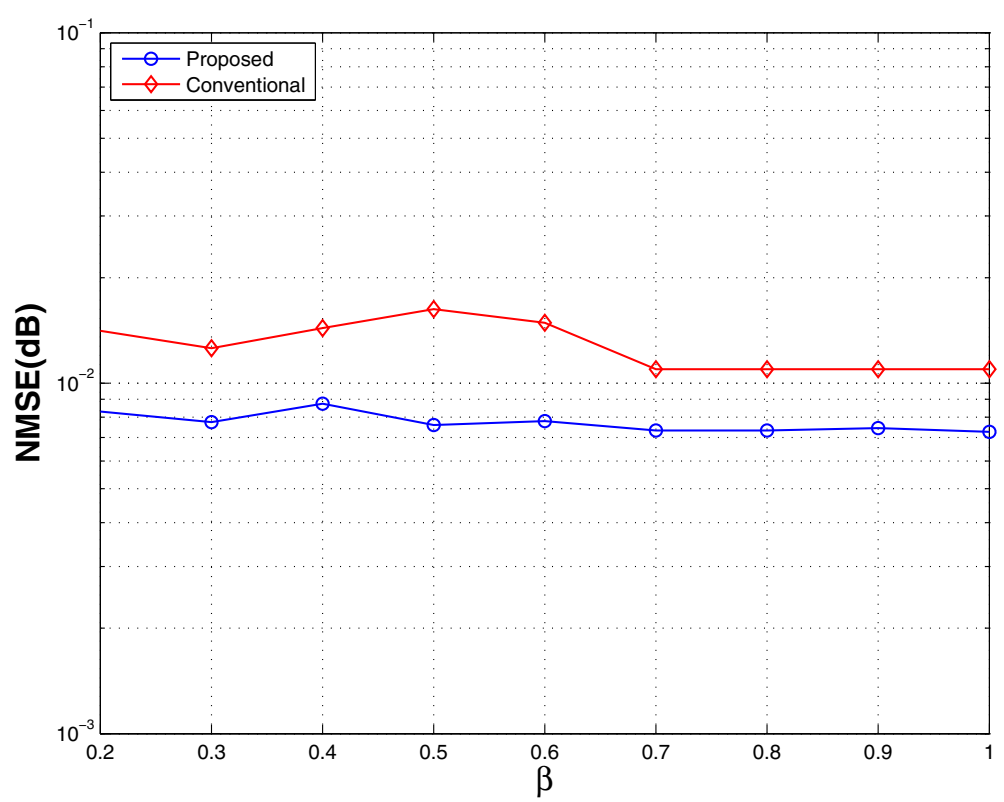

Figure 2 Comparison of the performance of the channel estimate MSE for different $\beta$ for $N=256$ and $L=N_{p}=8$. 


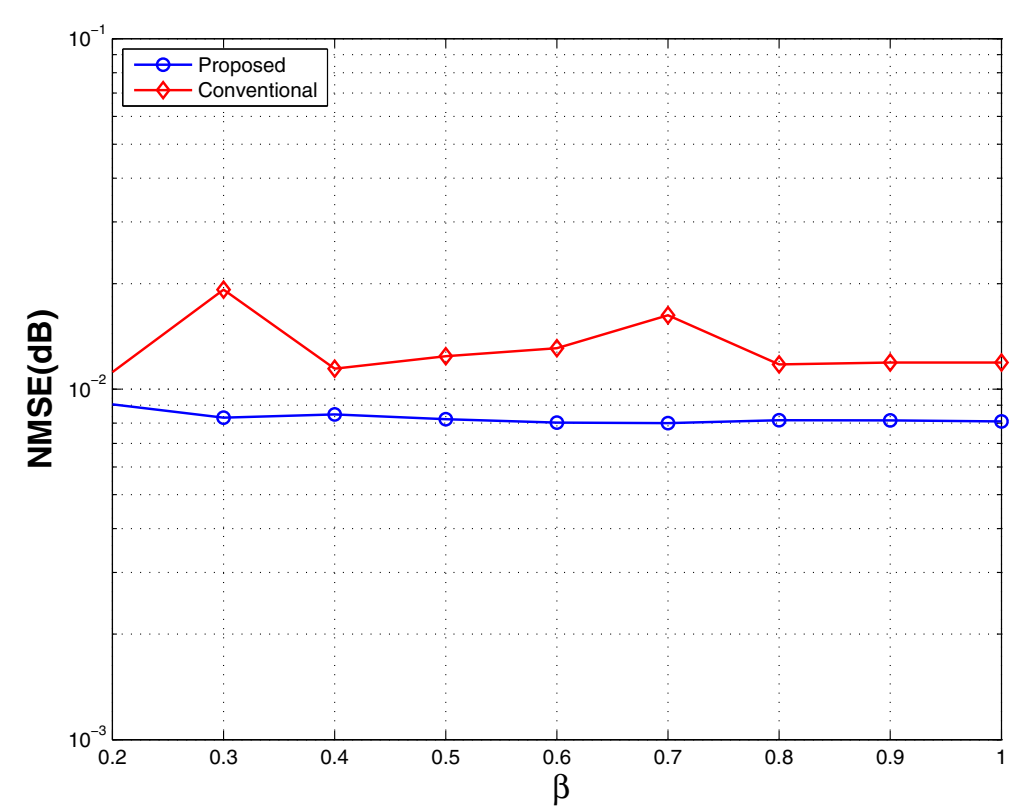

Figure 3 Comparison of the performance of the channel estimate MSE for different $\beta$ for $N=1024$ and $L=N_{p}=16$.

considered. This result demonstrates the effectiveness of our proposed scheme that optimizes both the position and power of the pilot symbols.

To further demonstrate the effectiveness of the proposed designs, we compare the MSE performance for different values of $\beta$ (i.e., different proportions of $|\mathcal{K}|$ and $N_{a}$ ). Figures 2 and 3 depict the MSE performance of the proposed method and the conventional equal powered pilot scheme for various $\beta$ when $\mathrm{SNR}=25 \mathrm{~dB}$.

From the result it is clear that the proposed design outperforms the conventional designs for all tested values of $\beta$. This substantiate the efficacious of the proposed scheme in designing pilot symbols for various sizes of the spectrum assigned to the cognitive SUs. Note that,

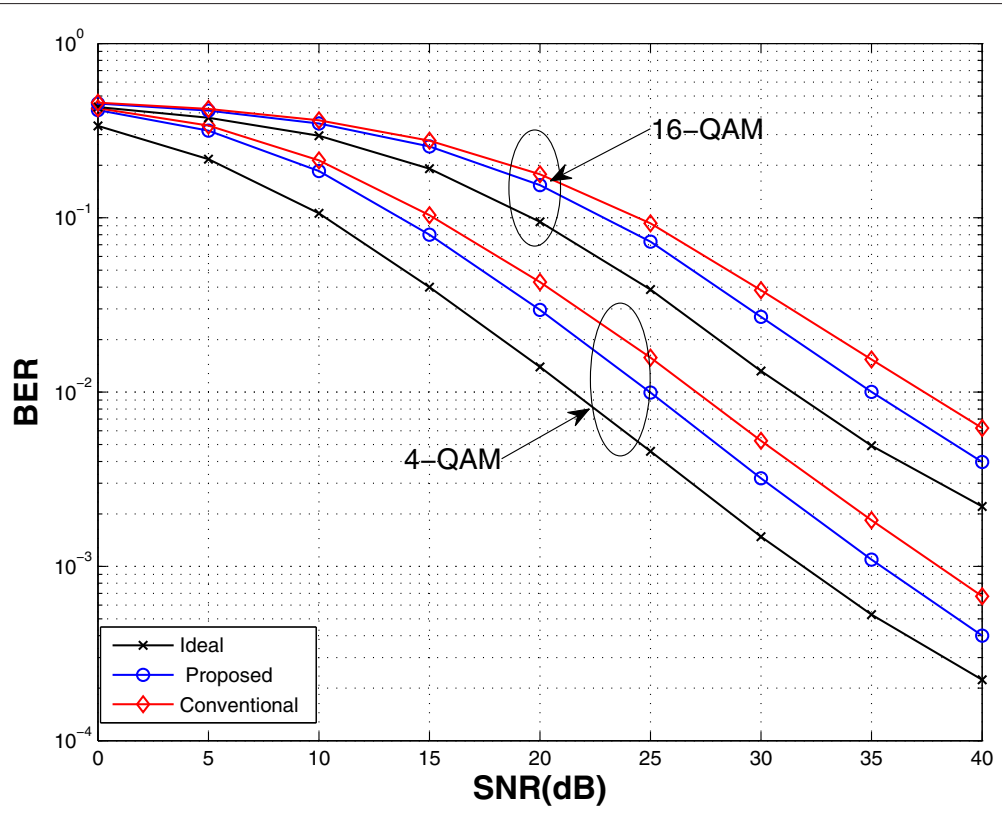

Figure 4 Comparison of the BER performances for QAM signals for $N=1024, L=N_{p}=16$ and $\beta=0.5$. 


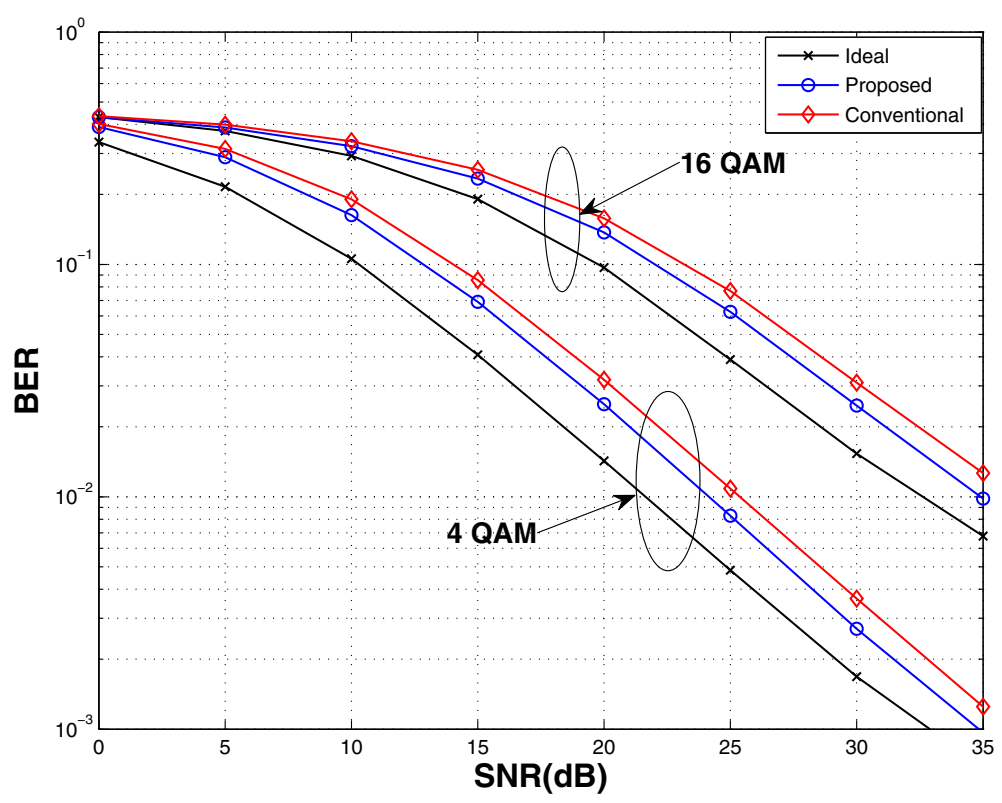

Figure 5 Comparison of the BER performances for QAM signals for $N=256, L=N_{p}=8$ and $\beta=0.5$.

the portions of the spectrum activated (or activated subcarriers) for the SUs are randomly generated for each value of $\beta$.

Next, we make comparison of the BER performance of the proposed pilot symbols, the conventional design in $[7,8]$ and the ideal case, i.e., known channel state information (CSI). The frequency-selective channel with $L=N_{p}$ taps is considered. Each channel tap is i.i.d. complex Gaussian with zero mean and the exponential power delay profile is given by the vector $\boldsymbol{v}=\left[v_{0} \cdots v_{L-1}\right]$, where $v_{l}=$ $\zeta e^{-l / 2}$, and $\zeta$ is a constant selected such that $\sum_{l=0}^{L-1} \nu_{l}=1$.

Figures 4 and 5 show BER performance of three schemes. The results show improved BER performance of the proposed design over the conventional design. This

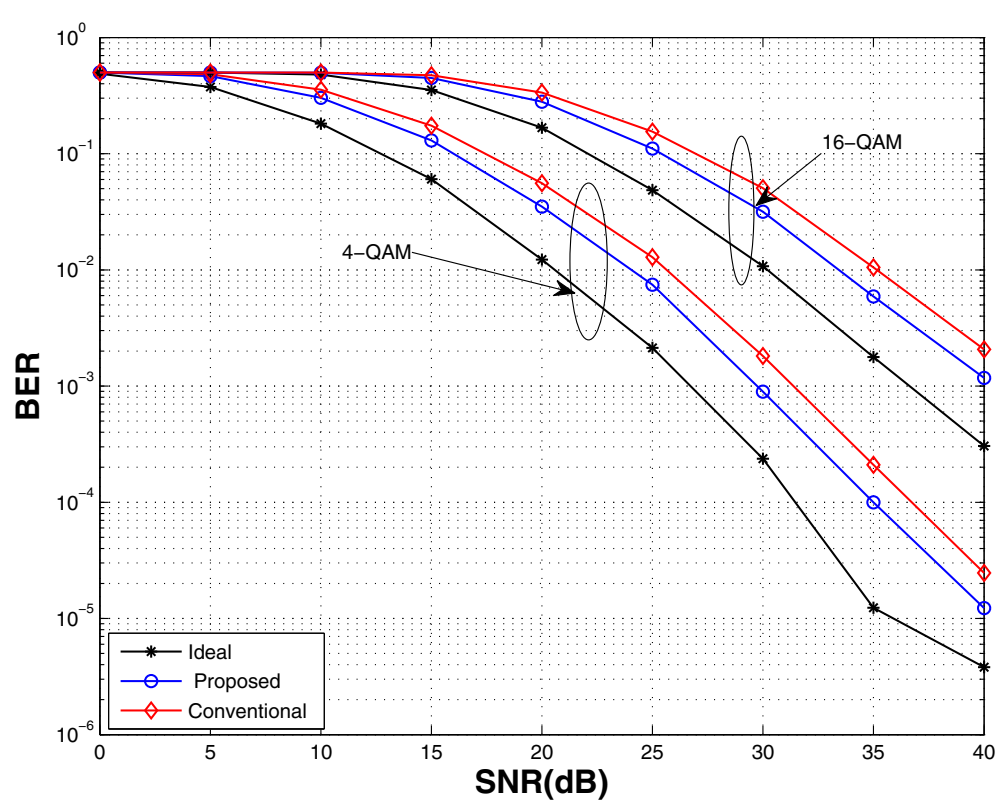

Figure 6 Comparison of the coded BER performances for QAM signals for $N=256, L=N_{p}=16$ and $\beta=0.5$. 
Table 1 Comparison of computational complexity between the CE, conventional and the exhaustive search scheme

\begin{tabular}{|c|c|c|c|c|c|c|}
\hline \multicolumn{7}{|c|}{ Computational complexity analysis } \\
\hline$\left(N_{d}, N_{p}\right)$ & $\mathcal{U}$ & $\mathcal{J}$ & $\begin{array}{l}\beta \\
|\mathcal{K}| / N_{a}\end{array}$ & $\begin{array}{l}\text { Conventional method } \\
\mathcal{O}\left(N_{a}^{2}+N_{a} N_{p}\right)\end{array}$ & $\begin{array}{l}\text { CE method } \\
\mathcal{O}\left(\left[2 * N_{d} N_{p}+N_{p} L\right] \mathcal{U} \mathcal{J}\right)\end{array}$ & $\begin{array}{l}\text { Exhaustive search method } \\
\mathcal{O}\left(\left[2 * N_{d} N_{p}+N_{p} L\right] \mathcal{U}\left(\begin{array}{c}|\mathcal{K}| \\
N_{p}\end{array}\right)\right)\end{array}$ \\
\hline$(32,8)$ & 200 & 5 & 0.2 & $1.92 \times 10^{3}$ & $5.76 \times 10^{5}$ & $4.43 \times 10^{10}$ \\
\hline$(24,16)$ & 200 & 5 & 0.2 & $2.24 \times 10^{3}$ & $1.0 \times 10^{6}$ & $6.44 \times 10^{13}$ \\
\hline$(92,8)$ & 200 & 5 & 0.5 & $1.08 \times 10^{4}$ & $1.54 \times 10^{6}$ & $2.86 \times 10^{14}$ \\
\hline$(84,16)$ & 200 & 5 & 0.5 & $1.16 \times 10^{4}$ & $2.90 \times 10^{6}$ & $3.96 \times 10^{21}$ \\
\hline 164,8 & 400 & 5 & 0.2 & $3.1 \times 10^{4}$ & $5.78 \times 10^{6}$ & $4.33 \times 10^{16}$ \\
\hline 156,16 & 400 & 5 & 0.2 & $3.23 \times 10^{4}$ & $1.05 \times 10^{7}$ & $7.17 \times 10^{25}$ \\
\hline 424,8 & 400 & 5 & 0.5 & $1.90 \times 10^{5}$ & $6.85 \times 10^{6}$ & $1.93 \times 10^{20}$ \\
\hline 416,16 & 400 & 5 & 0.5 & $1.94 \times 10^{5}$ & $1.36 \times 10^{7}$ & $7.2 \times 10^{32}$ \\
\hline
\end{tabular}

further substantiates the importance of optimizing both the pilot power and pilot positions in the NC-OFDM based systems.

Next we evaluate the BER performance when channel coding techniques are employed. Channel coding protects the data from errors by selectively introducing redundancies in the transmitted data. Convolutional encoding with Viterbi decoding, which is one of the forward error correction (FEC) techniques is considered. Interleaving and deinterleaving techniques are employed to reduce burst errors in transmitted data. Figure 6 depicts the performance of the coded BER of the three schemes over frequency selective channel. The FEC code used in the simulation is a simple symbol rate $1 / 2$, convolutional encoder $(171,133)$ with hard decision decoding. The results suggest that, the performance is mainly dictated by the accuracy of the estimated channel. Our proposed method outperforms the conventional method by a considerable margin. This suggests that, by employing FEC codes substantial coding gain may be attained and thereby provide better BER performance compared to the uncoded (raw) BER performance. However, the accuracy of the estimated channel still determine the best performing design.

We also compare the computational complexity in terms of the number of function evaluations for the proposed $\mathrm{CE}$, conventional and the exhaustive search (ES) scheme. Table 1 shows the complexity of the three schemes. Note that in all case we set $L=N_{p}$. It is clear that the conventional methods has lower complexity among the three algorithms, since it does not take power distribution into account. The complexity of the CE design is higher than that of the conventional reference scheme but relatively lower than that of the exhaustive search scheme. Also the complexity of the CE algorithm does increase rapidly with the increased number of data and pilot subcarriers. This suggests that $\mathrm{CE}$ algorithm is a prominent candidate when the solution space is large. Although the conventional scheme has lower complexity, its performance is inferior to that of the proposed scheme. There is a trade-off between performance and complexity, for applications that demands high performance, CE scheme can be a better compromise solution than the conventional method.

\section{Conclusions}

In this article, we have presented a new pilot symbol design for channel estimation in NC-OFDM cognitive radio systems. We have demonstrated that, for NC-OFDM based systems, to obtain better performance call for optimization of both pilot placement as well as pilot power distribution. We have also verified that, the improved performance obtained by optimizing the placement and power distribution of the pilot symbols is achieved at the expense of increased computational complexity. Simulation results show that, both MSE and BER performance of the proposed scheme outperforms the conventional equal powered pilot design. The complexity of our proposed design is slightly higher than that of the conventional scheme but combatively lower than that of the exhausted search scheme.

\section{Competing interests}

The authors declare that they have no competing interests.

Received: 20 June 2012 Accepted: 25 February 2013

Published: 16 March 2013

\section{References}

1. SEW Group, Spectrum Policy Task Force. Tech. Rep. FCC ET Docket 02-155, Federal Communications Commission (2002)

2. SM Mishra, D Cabric, C Chang, D Willkomm, B van Schewick, S Wolisz, BW Brodersen, in Proc. First IEEE Int. Symp. New Frontiers in Dynamic Spectrum Access Networks DySPAN. A real time cognitive radio testbed for physical and link layer experiments (Baltimore, MD, USA, 2005), pp. 562-567

3. G Bansal, MJ Hossain, VK Bhargava, Optimal and suboptimal power allocation schemes for OFDM-based cognitive radio. IEEE Trans. Wirel. Commun. 7(11), 4710-4718 (2008)

4. IJ Mitola, JGQ Maguire, Cognitive radio: making software radios more personal. IEEE Pers. Commun. 6(4), 13-18 (1999)

5. S Haykin, Cognitive radio: brain-empowered wireless communications. IEEE J. Sel. Area Commun. 23(2), 201-220 (2005) 
6. J Liu, S Feng, H Wang, in Proc. 5th Int. Conf. Wireless Communications, Networking and Mobile Computing WiCom. Comb-type pilot aided channel estimation in non-contiguous OFDM systems for cognitive radio (Beijing, China, 2009), pp. 1-4

7. D Hu, L He, in Proc. IEEE Global Telecommunications Conf. (GLOBECOM). Pilot design for channel estimation in OFDM-based cognitive radio systems (Miami, FL, USA, 2010), pp. 1-5

8. D Hu, L He, X Wang, An efficient pilot design method for OFDM-based cognitive radio systems. IEEE Trans. Wirel. Commun.

10(4), 1252-1259 (2011)

9. Z Hasan, G Bansal, E Hossain, V Bhargava, Energy-efficient power allocation in OFDM-based cognitive radio systems: A risk-return models. IEEE Trans. Wirel. Commun. 8(12), 6078-6088 (2009)

10. S Ohno, E Manasseh, M Nakamoto, Preamble and pilot symbol design for channel estimation in OFDM systems with null subcarriers. EURASIP. J. Wirel. Commun. Network. 2011, 1-17 (2011)

11. Q Huang, M Ghogho, S Freear, Pilot design for MIMO OFDM systems with virtual carriers. IEEE Trans. Signal Process. 57(5), 2024-2029 (2009)

12. RJ Baxley, JE Kleider, GT Zhou, Pilot design for OFDM with null edge subcarriers. IEEE Trans. Wirel. Commun. 8, 396-405 (2009)

13. BR Hamilton, X Ma, JE Kleider, RJ Baxley, OFDM pilot design for channel estimation with null edge subcarriers. IEEE Trans. Wirel. Commun. 10(10), 3145-3150 (2011)

14. L Tong, BM Sadler, M Dong, Pilot-assisted wireless transmissions: general model, design criteria, and signal processing. IEEE Signal Process. Mag. 21(6), 12-25 (2004)

15. S Adireddy, L Tong, H Viswanathan, Optimal placement of training for frequency-selective block-fading channels. IEEE Trans. Inf. Theory. 48(8), 2338-2353 (2002)

16. S Ohno, GB Giannakis, Capacity maximizing MMSE-optimal pilots for wireless OFDM over frequency-selective block Rayleigh-fading channels. IEEE Trans. Inf. Theory. 50(9), 2138-2145 (2004)

17. R Rubinstein, D Kroese, The Cross-Entropy Method: A Unified Approach to Combinatorial Optimization, Monte-Carlo Simulation and Machine Learning. (Springer- Verlag, New York, 2004)

18. R Rubinstein, The cross-entropy method for combinatorial and continuous optimization. Methodol. Comput. Appl. Probab. 2, 127-190 (1999)

19. W Maliki, Y Zhang, D O'Brien, D Edwards, Cross entropy optimization of MIMO capacity by transmit antenna selection. IET Microwave Antennas Propag. 1(6), 1131-1136 (2007)

20. E Manasseh, S Ohno, M Nakamoto, Design of low PAPR preamble and pilot symbol for channel estimation in OFDM systems. Int. J. Innov. Comput. Inf. Control. 7, 39-50 (2011)

21. E Manasseh, S Ohno, Y Jin, in The 2011 Asia-Pacific Signal and Information Processing Association Annual Summit and Conference (APSIPA ASC). Minimization of PAPR in MIMO-OFDM systems by tone reservation techniques and pilot tones (Xian, China, 2011), pp. 1-4

22. JC Chen, The cross-entropy method for maximum likelihood location estimation based on IEEE 802.15.4 radio. IEICE Trans. Commun. E91-B(8), 2724-2727 (2008)

23. Z Botev, D Kroese, An efficient algorithm for rare-event probability estimation, combinatorial optimization, and counting. Methodol. Comput. Appl. Probab. 10(4), 471-505 (2008)

24. E Manasseh, S Ohno, M Nakamoto, in European Signal Processing Conference. Pilot symbol design for channel estimation in MIMO-OFDM systems with null subcarriers (Aalborg, Denmark, 2010), pp. 1612-1616

25. IEEE Standard for Local and Metropolitan Area Networks Part 16: Air Interface for Fixed Broadband Wireless Access Systems (2004). [IEEE Std 802.16-2004]. http://ieeexplore.ieee.org/Xplore/defdeny.jsp?url= http://ieeexplore.ieee.org/stamp/stamp.jsp?tp=\&arnumber=
1350465\&userType=\&denyReason=-133\&arnumber= 1350465\&productsMatched=null\&userType=inst

26. IEEE Standard for Local and metropolitan area networks Part 16 Air Interface for Broadband Wireless Access Systems Amendment 3 : Advanced Air Interface (2011). [IEEE Std 802.16m-2011 (Amendment to IEEE Std 802.16-2009)]

doi:10.1186/1687-6180-2013-51

Cite this article as: Manasseh et al:: Pilot symbol assisted channel estimation for OFDM-based cognitive radio systems. EURASIP Journal on Advances in Signal Processing 2013 2013:51.

\section{Submit your manuscript to a SpringerOpen ${ }^{\mathcal{O}}$ journal and benefit from:}

- Convenient online submission

Rigorous peer review

- Immediate publication on acceptance

- Open access: articles freely available online

- High visibility within the field

- Retaining the copyright to your article

Submit your next manuscript at $\boldsymbol{\nabla}$ springeropen.com 